\title{
A MATHEMATICAL ANALYSIS OF THE INCLUSION OF INSTITUTIONAL BETTING FUNDS INTO STOCK MARKET: THE CASE OF TECHNICAL AND FUNDAMENTAL PAYOFF MODELS IN HORSERACE BETTING
}

\author{
Craig George Leslie Hopf and Gurudeo Anand Tularam \\ Science Environment Engineering and Technology (ENV), \\ Environment Future Centre, Griffith University, Australia
}

Received 2014-07-18; Revised 2014-07-29; Accepted 2014-08-13

\begin{abstract}
Horserace win and place returns have yet to be considered seriously as portfolio inputs in financial trading markets. However there exist technical and fundamental stochastic models of parametric and nonparametric distribution that appear to optimize expected returns from win and place investing in the horserace wagering market. In this study, a complex number optimization technique is introduced and applied to develop a deterministic betting model that calculates actual returns from the win and place betting. Using classes of models mentioned, field win bet payoff results were generated for a sample of successive global galloper races from Australasia, Asia and the United Kingdom. It was noted double digit returns exceeding $10 \%$ were consistently achieved within minutes of horserace investing, with arbitrage opportunity locked into prerace from the deterministic model. The results from this study provide evidence for serious investigation into the inclusion and benefit of Institutional Betting Funds into the local stock market.
\end{abstract}

Keywords: Models on Horserace Outcomes, Institutional Betting Funds, Stock Market, Horserace Betting

\section{INTRODUCTION}

'Financial markets are typically defined by having transparent pricing, basic regulations on trading, costs and fees and market forces determining the prices of securities that trade' (Investopedia, 2013). The horserace wagering market inherits uncertain investment returns, many participants and supply expansive information concerning participants and products typical of financial markets (Ali, 1998). Betting Market elements include institutional regulators (International Horseracing Federation), the participants (breeder, trainer, owner, jockey and bettor) and the betting products (win, place, quinella, trifecta). Global horserace annual wagering turnover is significant; for example, for year 2008 the turnover was AUD\$0.25 trillion (AGC, 2011), while theAustralian horserace annual turnover was AUD\$12.6 billion. The amount can be compared to the ASX equity annual turnover of AUD \$1.6 trillion (ASXG, 2008).

Global horserace wagering facilities have evolved from the track and Licensed Betting Office (LBO) outlets to online tote or fixed odd betting and person-to-person (p2p) betting exchange operations (Laffey, 2005). A betting exchange is an entity that provides trading facilities for retail or bookmaker customers to buy or sell contracts (Koning and Van Velzen, 2009). Horserace wager contracts are structured as binary options (typically European style) where the payoff is either some fixed amount from a win or place bet or nothing from a loss. One party is the layer (acceptor) of the bet and the counterparty are the bet taker. Betting exchange products include horserace wagering contracts, financial spread betting and contract for difference financial derivatives. The betting exchanges can be claimed to have brought transparency and trading

Corresponding Author: Craig George Leslie Hopf, Science Environment Engineering and Technology (ENV), Environment Future Centre, Griffith University, Australia 
innovation to horserace betting markets necessary for institutional investment (Laffey, 2005).

Betting activity on the exchange may be classified into speculative, hedging or arbitrage trading (Arnold, 2002). In 2011, the UK-founded Betfair betting exchange recorded an annual sale activity of 916 million bets matched; an average of 7 million transactions on the betting exchange daily. Moreover, Betfair recorded a new peak load of 30000 beats per minute during the 2011 Grand National steeplechase event (Betfair, 2011). Several factors appear to have contributed to Betfair's growth that include reduced online bettor transaction costs, the implication from Moore's Lawmore affordable computing gives firms and customers access to enormous processing power; and the adaptation from Metcalfe's Law of Networks that as the number of customers using Betfair multiplies, so does the utility of each customer. Others such as greater liquidity in the market and hence greater efficiency also aided Betfair's growth (Davies, 2005).

The Betfair model is based upon the open outcry system whereby backers and layers stakes are pool matched (Davies, 2005). Goldman Sach's technological division provides services concerning the development of both technical and fundamental model based algorithmic trading software. The models generate automatic execution strategies that can be used by the banker's internal equity businesses, as well as external clients such as fund managers and hedge funds (Goldman Sachs, 2011). Research in recent times has focused on optimal horserace betting models that forecast horserace outcomes based on both 'technical' and 'fundamental' analysis techniques (Edelman, 2007). The technical betting models utilize horserace market odds that are the bettor's probabilities with assumed market efficiency. This model quantifies both historical and current horserace variable data; while the fundamental modelling techniques utilize selective researched variables for race forecast, such as a Support Vector Machine/Conditional Logit (SVM/CL) horserace betting modelthat can generate double digit return trial results (Lessmann et al., 2009).

The main aim of this study is to develop and demonstrate a new technical deterministic betting model. The analysis will show that consistent profitable trading at an acceptable reward-to-risk level for the institutional betting fund can be achieved. The significance of deterministic models to lock in prerace guaranteed payoff for the fund is critically examined. In the first section a review of the existing literature on stochastic technical and fundamental rank order horserace betting models used to develop trading algorithms is presented. In the next section, a multiple system optimization theorem over the complex number field is presented and the deterministic horserace betting model developed from the theorem is used to generate returns from win and place betting. The model testing is then conducted on a random sample of global horseraces by simulating continuous trading and recording payoff results. The deterministic model payoff results are then compared with payoffs from selective technical stochastic models and the final section presents a discussion of the application of the new deterministic betting model in institutional betting fund trading.

\section{TECHNICAL AND FUNDAMENTAL STOCHASTIC BETTING MODELS}

\subsection{Racer Rank Notation}

The rank for the outcome of an event expressed by a rater is the result of ranking of a random variable from an underlying nonparametric or parametric distribution (D'Elia, 2003). Assigning the probability to the outcome from an ' $n$ ' field horserace is equivalent to assigning the probability for the rank permutation of the first ' $n$ ' racers. In this study, $\boldsymbol{X}_{\boldsymbol{i}}$ denotes an independent, non-identically distributed continuous random variable to represent time taken for the $i^{\text {th }}$ rank racer, with probability distribution $\boldsymbol{F}\left(\boldsymbol{X}_{i} ; \boldsymbol{\alpha}_{i}\right)$. The order of finish for a field size of ' $n$ ' racers is represented by the permutation $=(1,2, \ldots, n)$ whereby 1 represents the first placed racer and ${ }_{n}$ is the last placed racer from the field. The probability assigned to rank permutation is $p()=p\left({ }_{1}, 2, \ldots, n\right)=p\left(X_{R_{1}}<X_{R_{2}}<\ldots<X_{R_{n}}\right)$. This class of rank models is described as order statistics models that share the property that the order of any subset of the items is independent to the ordering of any disjoint subsets (Critchlow et al., 1991; Ali, 1998). Permutation group ${ }_{n}=$ \{\}$, n \varepsilon I$, represents the set of complete and partial rank order permutations for the ' $n$ ' racer field and $p()$ the parametric or nonparametric distribution on permutation $\mathrm{R}$.

The technical and fundamental models revised in this study share a multistage sequential process to generate permutation probability from bettor win odds to calculate expected payoff. For example, win( $\left(_{1}\right)$, quinella $\left({ }_{1}, 2\right)$ and trifecta $(\mathbf{1}, \mathbf{2}, \mathbf{3})$ illustrate betting market products. In particular, parametric distributions based upon the normal random variable $f\left(X_{i} ; \alpha_{i}, \sigma^{2}\right)$, the gamma random variable $f\left(X_{i} ; \alpha_{i}, \mathbf{r}\right)$ and the exponential random variable $f\left(X_{i} ; \alpha_{i}, \mathbf{r}=1\right)$ have been proposed for technical betting model application. These technical models share the Ldecomposability property that the probability attached to the ranking of a racer is independent from the relative 
ordering of the higher ranked racers (Critchlow et al., 1991). The fundamental betting models revised in this study utilize a two stage process to combine selective horserace variable data with the bettor odds variable to generate win (or place) probability. These include rank order Multiple Linear Regression/Conditional Logit (MLR/CL) and Conditional Logit/Conditional Logit (CL/CL) models and classifier Support Vector Machine/Conditional Logit (SVM/CL) model (Edelman, 2007; Lessmann et al., 2009).

\subsection{Technical Betting Model Optimization Techniques}

The ranking processes of the L-Decomposable models of Luce (1959; Harville, 1973; Hausch and Ziemba, 1985; Stern, 1990), all determine the conditional product of the choice probabilities (i.e., preferred racer from remaining racers) across the multistage sequence Equation 1:

$$
\begin{aligned}
& \left.\left.p()=p\left(1,2, \ldots,,_{n}\right)=\left\{p_{(1 . . n}\right)(1)\right\}\left\{p_{(2 . . n}\right)(2)\right\} . . \\
& \left.\left\{p_{(n-1 . . n}\right)\left({ }_{n-1}\right)\right\}
\end{aligned}
$$

$\boldsymbol{B}_{\boldsymbol{i}}=\left\{{ }_{i \bullet \bullet_{n}}\right\}$ is the set remaining at stage ' $\mathrm{i}$ '; with $\sum_{i=1}^{n} p\left({ }_{i}\right)=\mathbf{1}$. These multistage sequential processes utilize the bettor win odds $\boldsymbol{p}\left({ }_{i}=\mathbf{1}\right)=1 / O_{i}\left(O_{i}:\right.$ win odds $)$ to calculate win, place and compound betting products. The technical betting models attempt to achieve consistent profitable betting return, which challenges the semi-strong market efficient hypothesis that historical and publicly available horserace data has been correctly factored into the current bettor odds. The HZR wagering system calculates optimal place bets to maximize expected logarithm of final wealth from place wagering (Hausch and Ziemba, 1985). The model calculates the expected return from one additional dollar bet to decide racer selections against a breakeven benchmark and optimal bet sizes for the selected racers are calculated to maximize final wealth. The modern online betting portals should only enhance this model's performance from the presumption that pool wagering occurs just prior to the close of the betting period (Hausch and Ziemba, 1985). A modified version of the HZR model for algorithm development using bothwin and place totalizer bettor odds is as follows Equation

$E X_{i}^{P}=\sum_{j=1}^{n-1} \sum_{k=1}^{n-2}\left[\left\{\frac{w_{i} q_{j} q_{k}}{\left(1-q_{i}\right)\left(1-q_{i}-q_{j}\right)}\right\}+\left\{\frac{w_{j} q_{i} q_{k}}{\left(1-q_{j}\right)\left(1-q_{j}-q_{i}\right)}\right\}+\left\{\frac{w_{k} q_{j} q_{i}}{\left(1-q_{k}\right)\left(1-q_{j}-q_{k}\right)}\right\}\right]$

$\times\left[1+\frac{1}{20} I N T\left[\frac{Q(P+1)-\left(1+P_{i j k}\right)}{3\left(P_{i}+1\right)} \times 20\right]\right.$,

$E X_{i}^{P}$ : expected place return racer ' $i$ ',

$w_{i}$ : win odds racer ' $i$ '; $q_{i}$ : place odds racer ' $i$ ',

$E X_{i}^{P} \geq \gamma \geq 1, \gamma$ :breakeven benchmark parameter,

$\underset{\left\{p_{l}\right\}}{\operatorname{Maximize}} \operatorname{EX}\left(w_{f}\right)=\sum_{i=1}^{n} \sum_{j \neq i, j=1}^{n-1} \sum_{k \neq i, j ; k=1}^{n-2} \frac{w_{i} q_{j} q_{k}}{\left(1-q_{i}\right)\left(1-q_{i}-q_{j}\right)}$
$\times \log \left[\frac{Q\left(P+\sum_{l=1}^{n} p_{l}\right)-\left(P_{i j k}+p_{i j k}\right)}{3} \times\left[\frac{p_{i}}{P_{i}+p_{i}}+\frac{p_{j}}{P_{j}+p_{j}}+\frac{p_{k}}{P_{k}+p_{k}}\right]+w_{0}-\sum_{l=1}^{n} p_{l}\right]$,

EX $\left(w_{f}\right)$ : expected logarithm final wealth from place wagering,

$w_{0}$ : initial wealth; $Q$ : track payback,

$P$ : place pool, $P_{i j k}=P_{i}+P_{j}+P_{k}$,

$p_{l}$ : optimal place bets, $p_{i j k}=p_{i}+p_{j}+p_{k}$ 
The Logistic model generates permutation probabilities from the logarithm of win probability ratios (Plackett, 1975). The first-order logistic model corresponds to the L-Decomposable model,

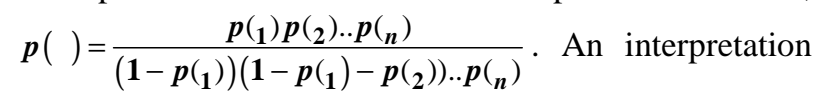
of the model is that a racer's rank is independent of earlier selections in accordance with the Ldecomposability property.

A second-order logistic model illustration for a four racer field has permutation probability (Dansie, 1983) Equation 3:

$$
\begin{aligned}
& p()=p\left[\left\{{ }_{12}<_{i j} \text { all } i, j \neq 1,2\right\} \text { and }\left\{{ }_{23}<_{24}\right\}\right], \\
& p()=p\left({ }_{1234}\right)=\frac{p(12) p(23)}{p\left({ }_{23}\right)+p\left({ }_{24}\right)}
\end{aligned}
$$

The probability association between racer pairs is considered by the second-order logistic model. A third-order logistic model illustration for a five racer field is $p()=\frac{p\left({ }_{123}\right) p\left({ }_{34}\right)}{p\left({ }_{34}\right)+p\left({ }_{35}\right)}$. A model derived from the extension of the L-Decomposable and gamma models is the discount model, which includes a discount factor $\lambda_{k}^{r}$ (decrease function as ' $k$ ' increases and dependent on shapeparameter ' $r$ ') to discount diminished racer performance with decrease in placing (Lo et al., 1995). The log odds ratio assumption that racer ' $i$ ' beats racer ' $j$ ' for $k^{\text {th }}$ place being a discounted function of racer ' $i$ ' defeating racer ' $j$ ' for the win, $L O(i, j \backslash k)=L O(i, j \backslash 1)^{\lambda_{k}^{r}}$. A discount model trifecta probability approximation is provided (Lo et al., 1995) Equation 4:

$$
\boldsymbol{p}\left({ }_{i j k}\right) \approx \boldsymbol{p}\left({ }_{i}\right) \frac{\left[\left(p\left({ }_{j}\right)\right)^{\lambda_{2}^{r}}\right]}{\left[\sum_{s \neq i}\left(p\left({ }_{s}\right)\right)^{\lambda_{2}^{r}}\right]} \frac{\left[\left(p\left({ }_{k}\right)\right)^{\lambda_{3}^{r}}\right]}{\left[\sum_{t \neq i, j}(p(t))^{\lambda_{3}^{r}}\right]}
$$

The discount model is a function of the win probabilities of all the racers in the field, unlike the LDecomposable model which is a function of the win probabilities of only selective racers. The inverse hypergeometric model similarly applies a sequential comparison criterion of bettor win or place odds to investigate ranking process outcomes (D'Elia, 2003).
The normal rank order model is a class of rank model that is a function of a single independent variable of parametric distribution $N\left(X_{i} ; \alpha_{i}\right)$ with joint pdf

$$
\prod_{i=1}^{n} \boldsymbol{f}\left(\boldsymbol{x}_{i}-\boldsymbol{\alpha}_{i}\right)\left(\alpha_{i} \text { : average timei }{ }^{\text {th }} \text { racer }\right) \text {. }
$$

Permutation probability for the normal rank model is represented by the multivariate integral Equation 5:

$$
\begin{aligned}
& p()=p(12 \cdot \cdot n) \equiv p\left(X_{R_{1}}<X_{R_{2}}<\ldots<X_{R_{n}}\right), \\
& =\int_{0}^{\infty} f\left(X_{1}-\alpha_{1}\right) \int_{X_{1}}^{\infty} f\left(X_{2}-\alpha_{2}\right) . . \int_{X_{n-1}}^{\infty} f\left(X_{n}-\alpha_{n}\right) \\
& d X_{1} . . d X_{n}\left(\sigma^{2}=1\right)
\end{aligned}
$$

The win racer ' $i$ ' probability for $\bar{X}_{\mathrm{R}}$ (expected win time)

is expressed $p\left({ }_{i}=1\right)=\int_{0}^{\bar{X}_{R}} f\left(X_{i} ; \alpha_{i}\right) \prod_{j \neq i=1}^{n-1}\left[1-F\left(X_{j}-\alpha_{j}\right)\right] d X_{i}$.

Henery (1981) derived the normal rank approximate model from first degree Taylor expansion to calculate the permutation probability as $p() \approx \frac{1}{n !}+\frac{\sum_{i=1}^{n} \alpha_{i} \mu_{i ; n}}{n !}\left(\sum \boldsymbol{\alpha}_{i}=\mathbf{0}\right)$. The win, place and trifecta probability approximations are illustrated by the following forms in Equation 6:

$$
\begin{aligned}
& p\left({ }_{i}=1\right) \approx \frac{1}{n}+\frac{\alpha_{i} \mu_{1 ; n}}{(n-1)}, \\
& p\left({ }_{j}=k\right) \approx \frac{1}{n}+\frac{\alpha_{j} \mu_{k ; n}}{(n-1)}, \\
& p\left({ }_{123}\right) \approx \frac{1}{n_{(3)}}\left[\begin{array}{l}
1+\sum_{i=1}^{3} \alpha_{i} \mu_{i ; n} \\
\sum_{j=1}^{3} \alpha_{j} \sum_{j=1}^{3} \mu_{j ; n} \\
(n-3)
\end{array}\right]
\end{aligned}
$$

The gamma rank order model class is a function of bivariate independent variables with gamma distribution $\Gamma\left(X_{i} ; \quad \alpha_{i}, \quad r\right)$ and joint pdf $\prod_{i=1}^{n}\left[\frac{\alpha_{i}^{r} X_{i}^{r-1} \exp \left(-\alpha_{i} X_{i}\right)}{\Gamma(r)}\right](\alpha:$ rate $; r:$ distance $)$.

The gamma rank permutation probability (Stern, 1990) is Equation 7: 
$p(1,2, \ldots, n) \approx \int_{0}^{X_{2}}\left(\frac{\alpha_{1}^{r} X_{1}^{r-1} \exp \left(-\alpha_{1} X_{1}\right)}{\Gamma(r)} \int_{0}^{X_{3}}\left(\frac{\alpha_{2}^{r} X_{2}^{r-1} \exp \left(-\alpha_{2} X_{2}\right)}{\Gamma(r)} \ldots \int_{0}^{\infty}\left(\frac{\alpha_{n}^{r} X_{n}^{r-1} \exp \left(-\alpha_{n} X_{n}\right)}{\Gamma(r)} d X_{1} . d X_{n}\right.\right.\right.$

And the racer probability to win (Henery, 1983) is $p\left({ }_{i}=1\right)=\int_{0}^{\infty} f\left(X_{i} ; \alpha_{i}, r\right) \prod_{j \neq i=1}^{n-1}\left[1-F\left(X_{j} ; \alpha_{j}, r\right)\right] d X_{i}$.

The gamma density function Equation 8:

$$
\begin{aligned}
& f\left(X_{i} ; \alpha_{i}, r\right)=\left(\frac{\alpha_{i}^{r} X_{r}^{r-1} \exp \left(-\alpha_{i} X_{i}\right)}{\Gamma(r)}\right) \\
& \equiv \exp \left\{-\alpha_{i} X_{i}+C\left(X_{i}\right)+D\left(\alpha_{i}\right)\right\}
\end{aligned}
$$

From which a first degree Taylor expansion approximation for the model is derived (Henery, 1983) as follows Equation 9:

$$
\begin{aligned}
& p() \approx p_{0}()+\sum_{i=1}^{n} \frac{\partial p()}{\partial \alpha_{i}}\left(\alpha_{i}-\alpha_{0}\right), \\
& p_{0}()=\frac{1}{n !}\left(\forall \alpha_{i}=\alpha_{0}\right), \\
& \frac{\partial p()}{\partial \alpha_{i}}=. . \frac{\partial}{\partial \alpha_{i}} \ln f\left(X_{i} ; \alpha_{i}, r\right) \prod_{i=1}^{n} f\left(X_{i} ; \alpha_{i}, r\right) d X_{i}, \\
& \frac{\partial p()}{\partial \alpha_{i}}=\frac{1}{n !}\left(-\mu_{i ; n}+D^{\prime}\left(\alpha_{i}\right)\right), \\
& p() \approx \frac{1}{n !}+\frac{\sum_{i=1}^{n}\left\{r-\mu_{i ; n}\right\}\left\{\alpha_{i}-1\right\}}{n !} \\
& \left(D\left(\alpha_{i}\right)=\ln \alpha_{i}^{r}, \alpha_{i} \rightarrow 1\right)
\end{aligned}
$$

A gamma rank probability approximation for the $k^{\text {th }}$ placed racer (Henery, 1983) is $p\left({ }_{i}=\boldsymbol{k}\right) \approx \frac{\mathbf{1}}{\boldsymbol{n}}+\frac{\left\{\boldsymbol{r}-\mu_{\boldsymbol{k} ; \boldsymbol{n}}\right\}\left\{\alpha_{i}-\mathbf{1}\right\}}{(\boldsymbol{n}-\mathbf{1})}$. The exponential model with density function $f\left(X_{i} ; \alpha_{i}\right)=\alpha_{i} \exp \left(-\alpha_{i} X_{i}\right)$ denotes the gamma rank order model with shape parameter $r=$ 1. The exponential model is equivalent to the LDecomposable model (Stern, 1990). The mathematical derivation of the L-Decomposable model from the gamma rank order model with shape parameter $r=1$, using conditional probability first principles is accordingly Equation 10:

$$
\begin{aligned}
& p\left(X_{1}=\min X_{j}\right) \\
& =\int_{0}^{\infty} \int_{X_{1}}^{\infty} \ldots \int_{X_{1}}^{\infty}\left[\prod_{j=1}^{n} \alpha_{j} e^{-\alpha_{j} X_{j}}\right] d X_{2} d X_{2} . . d X_{n}(r=1), \\
& p\left(X_{1}=\min X_{j}\right) \\
& =\int_{0}^{\infty} P\left(X_{j} \geq X_{1} \mid X_{1}\right) \alpha_{1} e^{-\alpha_{1} X_{1}} d X_{1} j=[2 . . n], \\
& p\left(X_{1}=\min X_{j}\right) \equiv \int_{0}^{\infty} \alpha_{1} e^{-\sum_{j=1}^{n} \alpha_{j} X_{1}} d X_{1}, \\
& p\left(X_{1}=\min X_{j}\right)=\frac{\alpha_{1}}{\sum_{j=1}^{n} \alpha_{j}} \\
& \therefore p()=p\left({ }_{1}, 2, \ldots, n\right)=\prod_{i=1}^{n-1}\left[\frac{p\left({ }_{i}\right)}{\sum_{j \varepsilon B_{i}} p\left({ }_{j}\right)}\right] ; B_{i}=\{i \cdot n\}
\end{aligned}
$$

These technical rank order models for horserace betting adopt permutation conditional probabilities and bettor odds to determine expected outcomes for typical horserace betting products, such as quinella, trifecta and first four horserace wagering. These elementary probabilities can be combined to optimize expected return from field betting.

\subsection{Fundamental Betting Model Optimization Techniques}

The fundamental model analysis utilizes relevant racer variables for win and place forecast based upon publicly available information. The predictive models that attempt to achieve a consistent profitable betting return do not satisfy the conditions of the semi-strong market efficiency theory that publicly available racer information has been factored into bettor odds (Lessmann et al., 2009). The inclusion of selective fundamental variables relevant to current and previous race information combined with current market odds data is one attempt to develop profitable stochastic models. CL/CL, MLR/CL and SVM/CL models possess such a two stage design; the first stage quantifies racer's ability based upon previous and current racer data and the second stage utilizes the bettor 
odds to provide consideration for within-race competition. The nonparametric MLR/CL technique models racer rank as a linear function of selective fundamental multivariable data to produce a winningness index forecast (score ability) in the first stage. Within-race competition is excluded from the first stage of the MLR/CL model but included in the second stage. Stage two develops a probability forecast for a racer win or place estimated in conjunction with competitors (within-race competition) by using a multinomial logit technique, which models a race as an entity; maintaining racer relationship and factoring in market bettor odds. The MLR/CL, CL/CL and $\mathrm{SVM} / \mathrm{CL}$ techniques differ in the first stage. The CL/CL model considers within competition with its modelling of publicly available racer information at stage one. The SVM/CL technique derives a classification model to identify race winners or losers and intentionally eliminating reliance upon rank order regression. The SVM/CL model utilizes a win or nonwin indicator variable rather than a finishing position in stage one (Lessmann et al., 2009). To construct nonlinear decision surfaces, support vector machine methodology map input fundamental data into a highdimensional feature space using a mapping function to minimize intensive calculation in the transformed feature space (Lessmann et al., 2009). Kernel functions can be employed to compute the scalar product of transformed vectors in the feature space. The Gaussian Radial Basis Function (RBF) kernel has been applied to horserace modelling with output values lying between zero and one (Edelman, 2007). The model below is a field win bet optimization strategy to determine optimal field bets to maximize expected logarithmic return and utilizing the MLR/CL two stage technique to forecast racer win probabilities. Stage one produces a winningness index as follows Equation 11:

$$
\begin{aligned}
& Y_{i j}=\beta_{0}+\beta_{1} X_{1}+\beta_{2} X_{2}+\ldots+\beta_{n} X_{n} \\
& +\mathcal{E} ; X_{1} . . X_{n}: \text { racer variables, } \\
& Y_{i j}=\frac{f p_{i j}}{(n+1)}-0.5 ; \sum_{j=1}^{n} Y_{i j}=0, Y_{i j} \in[-0.5,0.5], \\
& Y_{i j}: \text { normalized finish ( winningness index) racer } \\
& \quad ' j \text { ' in event ' } i \text { ', } \\
& f p_{i j}: \text { rank finish order racer ' } j \text { ' in event ' } i \text { '; } \\
& n: \text { number of racers in event ' } i \text { ' }
\end{aligned}
$$

The second stage of the MLR/CL model determines win probability forecasts for the individual racers Equation 12:

$L\left(\delta_{1}, \delta_{2}\right)$

$=\max \prod_{i=1}^{N \text { Races }} \frac{\prod_{j=1}^{n} \exp \left[\mu_{i j}\left(Y_{i j} \delta_{1}+\ln O_{i j} \delta_{2}\right)\right]}{\sum_{j=1}^{n} \exp \left(Y_{i j} \delta_{1}+\ln O_{i j} \delta_{2}\right)}$,

$\mathrm{L}\left(\delta_{1}, \delta_{2}\right)=\left(\hat{\delta}_{1}, \hat{\delta}_{2}\right):$ regularization parameter estimates,

$\mu_{i j}: \operatorname{binary}(0,1)$,

$p_{i j}=\frac{\exp \left(\hat{Y}_{i j} \hat{\delta}_{1}+\ln O_{i j} \hat{\delta}_{2}\right)}{\sum_{j=1}^{n} \exp \left(\hat{Y}_{i j} \hat{\delta}_{1}+\ln O_{i j} \hat{\delta}_{2}\right)}$,

$p_{i j}$ : probability racer ' $j$ ' wins event ' $i$ ',

$O_{i j}:$ bettor win odds racer ' $j$ ' wins event ' $i$ ',

$\boldsymbol{Y}_{i j}$ :winningness index forecast

The probability forecasts are combined with the bettor win odds to calculate optimal field bets to maximize expected logarithmic return (Edelman, 2007) in Equation 13:

$$
\begin{aligned}
& \underset{\left\{b_{i j}\right\}}{\text { Maximize }} \operatorname{EX}(R)=\sum_{j=1}^{n} p_{i j} \log \\
& {\left[b_{i j} O_{i j}-\left(\sum_{j=1}^{n} b_{i j}\right)\right]}
\end{aligned}
$$

EX $(R)$ : expected logarithm return,

$O_{i j}:$ bettor win odds;

$b_{i j}$ : optimal bet for racer ' $j$ 'event ' $i$ '

The stochastic fundamental and technical horserace betting market models, analogous to capital market models, optimize expected return on individual or field win and place assets. Furthermore, a property of parimutuel betting to beconsidered, is zero return - to - nil risk field betting is achievable from the gross bettor odds (before commission); subsequently positive actual returns can be achieved from favourable trading.

\section{COMPLEX NUMBER MULTIPLE SYSTEM OPTIMIZATION}

A set of algebraic elements and relations that define the set constitute a mathematical system. Complex and 
hypercomplex (quaternions, octonions) number systems are finite dimensional vector spaces over the real numbers that satisfy many of the real number system axioms. Complex analysis extends mathematical application beyond restrictions evident with the real number system's incapacity to describe all features of physical science. Consideration is given to horserace betting payoff represented by a complex number to separate field payoff from individual racer payoff. A deterministic model is one in which every set of variable states is uniquely determined by parameters in the model and by sets of previous states of these variables (Yang, 2008). In fact, deterministic modelling of future events through known parameters has significant application for financial market investment payoff, as evident in the capital debt markets. Schochetman and Smith (1998) develops an algorithm to generate average optimal solution in deterministic infinite horizon. The Multiple System Optimization (MSO) model is developed in this study.The model optimizes multiple complex system inputs, $\boldsymbol{Z}\left(\boldsymbol{C}^{n}\right.$;), over a finite horizon.

\section{Theorem 1}

Multiple System Optimization (MSO) over an ' $n$ ' finite series of complex systems generates a constant real component over each consecutive system in Equation 14:

$\{\max / \min \} Z_{1 . . n}\left(C_{1 . . n}^{n} ;\right)=g^{n}$,

$Z_{1 . . n}\left(C_{1}^{n} C_{2}^{n} . . C_{n}^{n}\right): n^{\prime}$ multiple system complex function,

$C^{n}$ : complex vector input; : set of operators,

${ }_{1 . . n}\left(\hat{C}_{1 . . n}^{n}\right):$ complex output $; \operatorname{Re}\left({ }_{1 . . n}\right)=g^{n}$

\section{Proof of Theorem 1}

The proof for the MSO theorem is by mathematical induction. The proof by induction involves a two stage process; firstly the base stage that is followed by the inductive stage. The base stage verifies that the optimization over a complex system, which comprises a complex vector argument and accompanying relations, generates a $Я$ real constant value. The inductive stage verifies that for a finite series of ' $n$ ' consecutive complex systems, the optimal solution is $Я^{n}$ for the multiple system complex function.

Maximizing or minimizing on the space $C^{n}$ of n-tuples of complex numbers must satisfy the n-dimensional Cauchy-Riemann equations in order for the complex function to be complex differentiable Equation 15:

$$
\begin{aligned}
& \boldsymbol{Z}\left(\boldsymbol{C}^{\boldsymbol{n}} ;\right)=\boldsymbol{Z}\left(\boldsymbol{C}_{\mathbf{1}}, \boldsymbol{C}_{\mathbf{2}}, \ldots, \boldsymbol{C}_{\boldsymbol{n}} ;\right) \mid C_{j}=\left(x_{j}, y_{j}\right), j \in\{1, \ldots, n\}, \\
& \frac{d Z}{d C}=\left[\frac{d Z}{d C_{1}}, \frac{d Z}{d C_{2}}, . ., \frac{d Z}{d C_{n}}\right], \\
& \frac{d Z}{d C}=\left[\frac{\partial Z}{\partial x_{1}} \frac{\partial x_{1}}{\partial C_{1}}, \frac{\partial Z}{\partial x_{2}} \frac{\partial x_{2}}{\partial C_{2}}, \ldots, \frac{\partial Z}{\partial x_{n}} \frac{\partial x_{n}}{\partial C_{n}}\right] \\
& +\left[\frac{\partial Z}{\partial y_{1}} \frac{\partial y_{1}}{\partial C_{1}}, \frac{\partial Z}{\partial y_{2}} \frac{\partial y_{2}}{\partial C_{2}}, . ., \frac{\partial Z}{\partial y_{n}} \frac{\partial y_{n}}{\partial C_{n}}\right], \\
& \frac{d Z}{d C}=\frac{1}{2}\left[\frac{\partial Z}{\partial x_{1}}-i \frac{\partial Z}{\partial y_{1}}, \frac{\partial Z}{\partial x_{2}}-i \frac{\partial Z}{\partial y_{2}}, . ., \frac{\partial Z}{\partial x_{n}}-i \frac{\partial Z}{\partial y_{n}}\right], \\
& \frac{\partial Z}{\partial x_{1}}=\frac{\partial Z}{\partial x_{2}}=, . .,=\frac{\partial Z}{\partial x_{n}}=0\{\text { Cauchy }- \text { Riemann }\}, \\
& \operatorname{Re}()=Я=\text { constant }
\end{aligned}
$$

Mathematical Induction step Equation 16:

$$
\begin{aligned}
& Z_{1 . . k}\left(C_{1}^{n} C_{2}^{n} . C_{k}^{n} ;\right)=g^{k}=\left\{Z_{1}\left(C^{n} ;\right)\right\}^{k}\{n=k\}, Z_{1 . . k+1} \\
& =Z_{1 . k}\left(C_{1}^{n} C_{2}^{n} . . C_{k}^{n} ;\right) Z_{1}\left(C^{n} ;\right),=\left\{Z_{1}\left(C^{n} ;\right)\right\}^{k} Z\left(C^{n} ;\right) \\
& =g^{k} g=g^{k+1}=Z_{1 . k+1}\left(C_{1}^{n} C_{2}^{n} . . C_{k+1}^{n} ;\right)\{Q E D\}
\end{aligned}
$$

\subsection{Deterministic Betting Model: Application of MSO}

It would seem that a deterministic model would be relevant for modelling betting market payoffs. The betting market assets (wager) possess defined payoffs with limited liability that is in contrast to equity and derivative market asset forecast payoff that are determined from the systematic and diversifiable factors (Williams, 1999). A zero risk horserace field betting provides an arbitrage opportunity for the trader and institutional betting fund. Optimization of the complex, nonlinear payoff function provided in Equation 17 determines racer field win and place returns from the available bettor fixed and totalizer odds:

$\{\max \} \operatorname{Payoff}\left(b^{n}, O^{n} ;\right)=\sum_{i=1}^{n}\left|O_{i} b_{i}\right|-\sum_{i=1}^{n} b_{i}$,

subject to $\{\min \}\left(b_{1} b_{2} . b_{n}\right)$,

$\left|\boldsymbol{O}_{i} \boldsymbol{b}_{\boldsymbol{i}}\right|=\left|\boldsymbol{O}_{j} \boldsymbol{b}_{j}\right|, \forall i \neq j$,

: set of operators, $b_{i}$ : betting amount $\left(b^{n} \in C^{n}\right)$,

$O_{i}:$ win / place odds $\left(O^{n} \in R^{n}\right)$ 
C.G.L. Hopf and G.A. Tularam / Journal of Mathematics and Statistics 10 (3): 390-400, 2014

Table 1. Trackinvest $\bigcirc$ model-win and place payoff race results: $R\left(R_{1} R_{2} R_{3}\right)=(12,3,4)$; win payoff $\mathbf{Z}\left(\mathbf{R}_{1}\right)=\{=30.30 \%$; place payoff $\mathbf{Z}\left(\mathbf{R}_{\mathbf{1}} \mathbf{R}_{\mathbf{2}} \mathbf{R}_{\mathbf{3}}\right)=$ Я $=5.9 \%$

\begin{tabular}{|c|c|c|c|c|c|c|c|c|c|c|c|}
\hline $\begin{array}{l}\text { Racer } \\
\left(\mathrm{R}_{\mathrm{i}}\right)\end{array}$ & $\begin{array}{l}\text { Mkt } \\
\text { odds }\end{array}$ & Bet & Win $\left(R_{i}\right)$ & $\begin{array}{l}\text { Net } \\
\text { return }\end{array}$ & Payoff & $\begin{array}{l}\text { Racer } \\
\left(\mathrm{R}_{\mathrm{i}}\right)\end{array}$ & $\begin{array}{l}\text { Mkt } \\
\text { odds }\end{array}$ & Bet & $\operatorname{Place}\left(\mathrm{R}_{\mathrm{ijk}}\right)$ & $\begin{array}{l}\text { Max-min } \\
\text { return }\end{array}$ & $\begin{array}{l}\text { Payoff } \\
\text { (max-min) }\end{array}$ \\
\hline 1 & 17.8 & $\$ 12000$ & $\$ 213600$ & $\$ 48600$ & $(29.45 \%, 0)$ & 1 & 3.4 & $\$ 21000$ & $\$ 71400$ & $(\$ 13600,-\$ 43600)$ & $(6.6 \%,-21.3 \%)$ \\
\hline 2 & 20.8 & $\$ 10000$ & $\$ 208000$ & $\$ 43000$ & $(26.06 \%, 0)$ & 2 & 3.6 & $\$ 20000$ & $\$ 72000$ & $(\$ 14200,-\$ 43000)$ & $(6.9 \%,-21 \%)$ \\
\hline 3 & 4.9 & $\$ 45000$ & $\$ 220500$ & $\$ 55500$ & $(33.64 \%, 0)$ & 3 & 1.6 & $\$ 45000$ & $\$ 72000$ & $(\$ 14200,-\$ 43000)$ & $(6.9 \%,-21 \%)$ \\
\hline 4 & 16.6 & $\$ 13000$ & $\$ 215800$ & $\$ 50800$ & $(30.79 \%, 0)$ & 4 & 7.3 & $\$ 10000$ & $\$ 73000$ & $(\$ 14200,-\$ 42000)$ & $(6.9 \%,-20.5 \%)$ \\
\hline 5 & 17.4 & $\$ 12000$ & $\$ 208800$ & $\$ 43800$ & $(26.55 \%, 0)$ & 5 & 5.3 & $\$ 14000$ & $\$ 74200$ & $(\$ 14200,-\$ 40800)$ & $(6.9 \%,-19.9 \%)$ \\
\hline 6 & 0.0 & & & & & 6 & 0.0 & & & & \\
\hline 7 & 0.0 & & & & & 7 & 0.0 & & & & \\
\hline 8 & 4.7 & $\$ 47000$ & $\$ 220900$ & $\$ 55900$ & $(33.88 \%, 0)$ & 8 & 1.9 & $\$ 37000$ & $\$ 70300$ & $(\$ 12500,-\$ 44700)$ & $(6.1 \%,-21.8 \%)$ \\
\hline 9 & 2.4 & $\$ 0$ & $\$ 0$ & $-\$ 165000$ & $(0 \%,-100 \%)$ & 9 & 1 & $\$ 20000$ & $\$ 20000$ & $(-\$ 37800,-\$ 44700)$ & $(-18 \%,-21.8 \%)$ \\
\hline 10 & 13.5 & $\$ 16000$ & $\$ 216000$ & $\$ 51000$ & $(30.91 \%, 0)$ & 10 & 3.5 & $\$ 20000$ & $\$ 70000$ & $(\$ 12200,-\$ 44700)$ & $(6 \%,-21.8 \%)$ \\
\hline 11 & 0.0 & & & & & 11 & 0.0 & & & & \\
\hline 12 & 21.5 & $\begin{array}{l}\$ 10000 \\
\$ 165000\end{array}$ & $\$ 215000$ & $\$ 50000$ & $(30.30 \%, 0)$ & 12 & 4 & $\begin{array}{l}\$ 18000 \\
\$ 205000\end{array}$ & $\$ 72000$ & $(\$ 14200,-\$ 43000)$ & $(6.9 \%,-21 \%)$ \\
\hline
\end{tabular}

source: Unitab - Riccarton ZS1 (24 February 2012)

Table 2. Trackinvest $\odot$ model-multibet win payoff racer results: $R^{1}\left(R_{1} R_{2} R_{3}\right)=(12,3,4), R^{2}\left(R_{1} R_{2} R_{3}\right)=(1,11,12)$; win payoff $Z_{1 . .2}$ $=\left(\mathrm{R}^{1} \mathrm{R}^{2}\right)=(1+Я)^{2}=(1+0.3030)(1+0.09)=1.42$, A $=1.42^{0.5}-1=19 \%$

\begin{tabular}{|c|c|c|c|c|c|c|c|c|c|c|c|}
\hline $\begin{array}{l}\text { Racer } \\
\left(\mathrm{R}_{\mathrm{i}}\right)\end{array}$ & $\begin{array}{l}\text { Mkt } \\
\text { Odds }\end{array}$ & Bet & $\begin{array}{l}\text { Win } \\
\left(\mathrm{R}_{\mathrm{i}}\right)\end{array}$ & $\begin{array}{l}\text { Net } \\
\text { return }\end{array}$ & Payoff & $\operatorname{Racer}\left(\mathrm{R}_{\mathrm{i}}\right)$ & $\begin{array}{l}\text { Mkt } \\
\text { odds }\end{array}$ & Bet & $\operatorname{Win}\left(\mathrm{R}_{\mathrm{i}}\right)$ & $\begin{array}{l}\text { Net } \\
\text { return }\end{array}$ & Payoff \\
\hline 1 & 17.8 & $\$ 12000$ & $\$ 213600$ & $\$ 48600$ & $(29.45 \%, 0)$ & 1 & 4.4 & $\$ 12000$ & $\$ 52800$ & $\$ 4500$ & $(9 \%, 0)$ \\
\hline 2 & 20.8 & $\$ 10000$ & $\$ 208000$ & $\$ 43000$ & $(26.06 \%, 0)$ & 2 & 0.0 & & & & \\
\hline 3 & 4.9 & $\$ 45000$ & $\$ 220500$ & $\$ 55500$ & $(33.64 \%, 0)$ & 3 & 6.7 & $\$ 7500$ & $\$ 50250$ & $\$ 1950$ & $(4 \%, 0)$ \\
\hline 4 & 16.6 & $\$ 13000$ & $\$ 215800$ & $\$ 50800$ & $(30.79 \%, 0)$ & 4 & 9.3 & $\$ 5500$ & $\$ 51150$ & $\$ 2850$ & $(6 \%, 0)$ \\
\hline 5 & 17.4 & $\$ 12000$ & $\$ 208800$ & $\$ 43800$ & $(26.55 \%, 0)$ & 5 & 10.7 & $\$ 4600$ & $\$ 49220$ & $\$ 920$ & $(2 \%, 0)$ \\
\hline 6 & 0.0 & & & & & 6 & 26.0 & $\$ 2000$ & $\$ 52000$ & $\$ 3700$ & $(8 \%, 0)$ \\
\hline 7 & 0.0 & & & & & 7 & 18.0 & $\$ 2800$ & $\$ 50400$ & $\$ 2100$ & $(4 \%, 0)$ \\
\hline 8 & 4.7 & $\$ 47000$ & $\$ 220900$ & $\$ 55900$ & $(33.88 \%, 0)$ & 8 & 3.2 & $\$ 4000$ & $\$ 12800$ & $-\$ 35500$ & $(0 \%,-73 \%)$ \\
\hline 9 & 2.4 & $\$ 0$ & $\$ 0$ & $-\$ 165000$ & $(0 \%,-100 \%)$ & 9 & 16.6 & $\$ 3000$ & $\$ 49800$ & $\$ 1500$ & $(3 \%, 0)$ \\
\hline 10 & 13.5 & $\$ 16000$ & $\$ 216000$ & $\$ 51000$ & $(30.91 \%, 0)$ & 10 & 50.6 & $\$ 1000$ & $\$ 50600$ & $\$ 2300$ & $(5 \%, 0)$ \\
\hline 11 & 0.0 & & & & & 11 & 19.6 & $\$ 2600$ & $\$ 50960$ & $\$ 2660$ & $(6 \%, 0)$ \\
\hline \multirow[t]{2}{*}{12} & 21.5 & $\$ 10000$ & $\$ 215000$ & $\$ 50000$ & $(30.30 \%, 0)$ & 12 & 14.9 & $\$ 3300$ & $\$ 49170$ & $\$ 870$ & $(2 \%, 0)$ \\
\hline & & $\$ 165000$ & & & & & & $\$ 48300$ & & & \\
\hline
\end{tabular}

Source: Unitab-Riccarton ZS1 and ZS2 (24 February 2012)

Table 1 and 2 illustrate field win and place payoff results generated from algorithm, which uses online or fixed horserace bettor odds and attempts to lock in a pre-race positive optimal payoff over the racer field for a total minimum investment. The complex payoff is determined using the MSO-the derived algorithm displays a return-to-risk trade-off that typifies financial market investments. This technical deterministic betting model's output separates field payoff from individual racer payoff. The return-to-risk trade-off shown in Table 1 is to lock in a pre-race $30 \%$ win payoff over the racer field, excluding the favourite, for the shortterm investment period (minutes). The risk is a loss from the favourite racer winning. An optional feasible trade-off is locking into the lower place payoff and lesser absolute maximum risk.
A trader could source the global market of betting exchanges and bookmakers for preferred win or place odds. Bet taking win market odds of 4.7 for the favourite would have achieved arbitrage and a 3\% payoff over the entire field. Table 2 displays the return-to-risk trade-off from multiple betting over two consecutive races. The horserace results shown in Table 2 generated a feasible payoff of $19 \%$ for the fund from consecutive race investing.

\section{4, DETERMINISTIC VERSUS STOCHASTIC BETTING PAYOFF- ANALYSIS AND RESULTS}

A sample of fifty consecutive galloper races for January 012012 from global racetracks of Australasia 
(Australia and New Zealand), Asia (Singapore and Hong Kong) and Great Britain, provided the data (http://www.sportsbet.com.au/results/horse_racing)for testing the performance and comparison of the technical deterministic and stochastic betting models in this study. The data gathered simulates "passing the bet global"continuous trading on betting market races from the southern to the northern hemisphere. Although dated, both Lo et al. (1995) and Ali (1998) tests show that the normal model forecasted horserace rank probabilities more accurately than the gamma rank or Ldecomposable models. Based on the data, the racer field win betting payoff from the deterministic model is compared with payoff from the normal rank approximate and L-decomposable models. The deterministic model optimizes racer field payoff $(Я)$-to-risk trade-off for minimum betting amount on consecutive races; zero risk reflects arbitrage opportunity and locks in a pre-race positive return independent of winning racer outcome. Both the normal approximate and L-decomposable stochastic models recorded the same expected payoff results. The net expected win return per $\$ 1$ unit bet from a winning racer for both the L-decompose and normal approximate models equals the negative of the tote track take $(\mathrm{t})$; and the net expected field win payoff for both models is $E$ (payoff $)=-(n+t-1) ; n$ : field size, $t$ : track take.

Table 3 displays model's results for the Australasian, Asian and UK sampled regions. Individual and accumulative race returns generated from both the DBM and the normal approximate technique are displayed. The DBM provide two payoffs: (a) A nil trade strategy where maximum exposure from an individual racer losing is $100 \%$; and (b) a case where the maximum risk of loss from an individual racer is capped at a maximum of $10 \%$. Uniform unit bets on the field for the normal approximate model resulted in sample payoff comparison with the DBM payoff results. Table 3 results demonstrate the superiority of the DBM over the stochastic model. The DBM consistently generated double digit bettor race return. Similar to the stochastic model results, the accumulative payoff generated from the DBM over a series of races is affected when a loss occurs with high exposure. Interestingly however, the DBM optimizing field win bets with betting exchange trade to maximize exposure to $10 \%$, resulted in both exceptional positive accumulative payoffs $(1+\mathscr{A})^{n}$ and successive racer return $Я$ achieved for the series of races in all the regions.

Table 3. Technical models-field win bet payoff

\begin{tabular}{|c|c|c|c|c|c|c|c|c|c|c|}
\hline \multirow[b]{2}{*}{ Region } & \multicolumn{8}{|c|}{ DBM Results (\%) } & \multirow[b]{2}{*}{$(1+Я)^{\mathrm{n}}$} & \multirow[b]{2}{*}{ Я(\%) } \\
\hline & race 1 & race 2 & race 3 & race 4 & race 5 & race 6 & race 7 & race 8 & & \\
\hline \multirow[t]{2}{*}{ UK (Cheltenham) } & 1.82 & 59.36 & (a) -29.16 & 8.21 & 15.81 & & & & (a) 1.44 & (a) 7.6 \\
\hline & & & (b) -10 & & & & & & (b) 1.83 & (b) 12.8 \\
\hline \multirow[t]{2}{*}{ SGP (Kranji) } & 7.87 & (a) -75.52 & & & & & & & (a) 0.382 & (a) -17.5 \\
\hline & & (b) -10 & 26.12 & 7.49 & 6.79 & & & & (b) 1.405 & (b) 7 \\
\hline \multirow[t]{2}{*}{ HKD (Sha Tin) } & 5.46 & (a) -62 & 17.46 & 6.55 & 11.98 & & & & (a) 0.562 & (a) -11 \\
\hline & & (b) -10 & & & & & & & (b) 1.33 & (b) 5.9 \\
\hline \multirow[t]{2}{*}{ AUS (Inverell) } & 12.62 & 9.08 & 4.44 & (a) -70.59 & 6.21 & 1.4 & (a) -78.46 & 6 & (a) 0.093 & (a) -26 \\
\hline & & & & (b) -10 & & & (b) -10 & & (b) 1.186 & (b) 2.16 \\
\hline \multirow[t]{2}{*}{ AUS (Mornington) } & 14.22 & 4.89 & 10.63 & 11.17 & (a) -81.46 & 32.01 & (a) -75.2 & (a) -76.98 & (a) 0.02 & (a) -39 \\
\hline & & & & & (b) -10 & & (b) -10 & (b) -10 & (b) 1.418 & (b) 4.46 \\
\hline \multirow[t]{2}{*}{ AUS (Murray Bridge) } & 31.78 & 16.48 & (a) -81.25 & 42.91 & 11.52 & (a) -65.87 & 6.69 & 10.09 & (a) 0.184 & (a) -19 \\
\hline & & & (b) -10 & & & (b) -10 & & & (b) 2.328 & (b) 11.14 \\
\hline \multirow[t]{2}{*}{ AUS (Sunshine Coast) } & (a) -25.4 & 52.28 & 3.17 & (a) -52.77 & 13.62 & (a) -71.33 & 0.32 & 2.64 & (a) 0.18 & (a) -19.3 \\
\hline & (b) -10 & & & (b) -10 & & (b) -10 & & & (b) 1.34 & (b) 3.73 \\
\hline \multirow[t]{3}{*}{ NZD (Waikouaiti) } & (a) -45.45 & 27.55 & (a) -72.86 & & & & & & (a) 0.19 & (a) -42.5 \\
\hline & (b) -10 & & (b) -10 & & & & & & (b) 1.033 & (b) 1.09 \\
\hline & \multicolumn{10}{|c|}{ Normal (approximate) Model Results (\%) } \\
\hline UK (Cheltenham) & -65 & 6.67 & -50 & -17 & 41.25 & & & & 0.219 & -26 \\
\hline SGP (Kranji) & -2.5 & -78 & 439 & -53.6 & -24.2 & & & & 0.407 & -16.5 \\
\hline HKD (Sha Tin) & 44.6 & -69.3 & 147.1 & -70 & 42.1 & & & & 0.468 & -14 \\
\hline AUS (Inverell) & -31.25 & 2.3 & -54.3 & -74.5 & -60.8 & 21.1 & -78.5 & 21.4 & 0.01 & -44 \\
\hline AUS (Mornington) & -56.25 & -15.6 & -46.4 & 8.6 & -69.2 & -37.5 & -78.3 & -67.8 & 0.003 & -52 \\
\hline AUS (Murray Bridge) & 25.7 & 238.2 & -66 & 8.3 & -30 & -64.3 & 1.7 & 62.9 & 0.65 & -5 \\
\hline AUS (Sunshine Coast) & -82.9 & -33.3 & -48 & -74.3 & -11 & -69 & -63.1 & -54 & 0.0007 & -60 \\
\hline NZD (Waikouaiti) & -77.5 & 25 & -70 & & & & & & 0.084 & -56.2 \\
\hline
\end{tabular}

Source: NSW TAB (01 January 2012) (a) max risk 100\% (b) max risk 10\% 


\section{IMPLICATIONS FOR THE INSTITUTIONAL BETTING FUND}

The results show that the DBM can be applied to all classes of racing including galloper, trotter and greyhound. Clearly, the DBM optimizes the pre-race (or within race) payoff-to-risk trade-off for win or place field betting for selective races or consecutive race series over the finite time horizon. The algorithms of win and place combination are worthy of consideration to enhance payoff performance. The bet take and lay trade enables a risk-free payoff; that is, the betting exchanges trade bettor odds that offset the track commission to lock-in pre-race deterministic return. The consistently generated double digit returns from the DBM for the global galloper sample provides evidence for further and more in depth investigation of institutional betting fund participation and liquidity contribution into the financial markets; including future consideration for portfolio investment inclusion.

\section{CONCLUSION}

The findings of this study show that deterministic and stochastic financial market models may be used to determine the reward-to-risk trade-off for an investment. The stochastic technical and fundamental betting models provide racer win and place probabilities and expected payoffs, from historical and current race information. The analysis shows that the deterministic modelling of a limited payoff and limitedliability investment generates a "fixed" or a certain outcome. Moreover, the DBM's generate an actual return from win and place wagering. The MSO generated a constant real component over consecutive systems. The DBM developed based on this technique optimizes field win and place betting and separates the optimal field payoff from the individual racer payoff. The individual racer exposure is related to the track take, which reduces the true bettor odds. Both the DBM and trading in the betting market can offset the track commission to achieve racer return field coverage at an acceptable risk; therefore, clearly arbitrage is achieveable. The preliminary findings from DBM testing of a global horserace sample in this study has generated significant payoff at acceptable risk for the Institutional Betting Fund; as precursor to attaining results from larger racer sampling periods to challenge the existing semi-strong efficient market hypothesis toward horserace betting.

\section{REFERENCES}

AGC, 2011. A database on australia's gambling industry. Australasian Gaming Council.

Ali, M.M., 1998. Probability models on horse-race outcomes. J. Applied Stat., 25: 221-229. DOI: $10.1080 / 02664769823205$

Arnold, G., 2002. Corporate Financial Management. 2nd Edn., Financial Times/Prentice Hall, Harlow England.

ASXG, 2008. ASX Ltd Annual Report. Australia.

Betfair, 2011. Betfair Annual Report. London.

Critchlow, D.E., M.A. Fligner and J.S. Verducci, 1991. Probability models on rankings. J. Math. Psychol., 35: 294-318. DOI: 10.1016/00222496(91)90050-4

Dansie, B.R., 1983. A note on permutation probabilities. J. Royal Stat. Society, 45: 22-24.

Davies, M., L. Pitt, D. Shapiro and R. Watson, 2005. "Betfair.com: Five technology forces revolutionize worldwide wagering. Eur. Manage. J., 23: 533-541. DOI: 10.1016/j.emj.2005.09.008

D'Elia, A., 2003. Modelling ranks using the inverse hypergeometric distribution. Stat. Modell., 3: 6578. DOI: $10.1191 / 1471082$ X03st047oa

Edelman, D., 2007. Adapting support vector machine methods for horserace odds prediction. Ann. Operat. Res., 151: 325-336. DOI: 10.1007/s10479-006-0131-7

Goldman Sachs, 2011. Goldman sachs annual report. United States.

Harville, D.A., 1973. Assigning probabilities to the outcomes of multi-entry competitions. J. Am. Stat. Assoc., 68: 312-316. DOI: 10.1080/01621459.1973.10482425

Hausch, D.B. and W.T. Ziemba,1985. Transactions costs, extent of inefficiencies, entries and multiple wagers in a racetrack betting model. Manage. Sci., 31: 381-394. DOI: $10.1287 / \mathrm{mnsc} .31 .4 .381$

Henery, R.J., 1981. Permutation probabilities as models for horse races. J. Royal Stat. Society, 43: 86-91.

Henery, R.J., 1983. Permutation probabilities for gamma random variables. J. Applied Probab., 20: 822-834. DOI: $10.2307 / 3213593$

Investopedia, 2013. Financial market. United States.

Koning, R.H. and B. Van Velzen, 2009. Betting exchanges: The future of sports betting? Int. J. Sport Finance, 4: 42-62. 
Laffey, D., 2005. Entrepreneurship and Innovation in the UK Betting Industry: The rise of person-to-person betting. Eur. Manage. J., 23: 351-359. DOI: 10.1016/j.emj.2005.04.013

Lessmann, S., M. Sung and J.E. Johnson, 2009. Identifying winners of competitive events: A SVMbased classification model for horserace prediction. Eur. J. Operat. Res., 196: 569-577. DOI: 10.1016/j.ejor.2008.03.018

Lo, V.S.Y., J. Bacon-Shone and K. Busche, 1995. The application of ranking probability models to racetrack betting. Manage. Sci., 41: 1048-1059. DOI: $10.1287 / \mathrm{mnsc} .41 .6 .1048$

Luce, R.D., 1959. Individual Choice Behavior: A Theoretical Analysis. 1st Edn., Wiley, New York, pp: 153.
Plackett, R.L., 1975. The analysis of permutations. J. Royal Stat. Society, 24: 193-202.

Schochetman, I.E. and R.L Smith, 1998. Existence and discovery of average optimal solutions in deterministic infinite horizon optimization. Math. Operat. Res., 23: 416-432. DOI: 10.1287/moor.23.2.416

Stern, H., 1990. Models for distributions on permutations. J. Am. Stat. Assoc., 85; 558-564. DOI: $10.1080 / 01621459.1990 .10476235$

Williams, L.V., 1999. Information efficiency in betting markets: A survey. Bull. Econom. Res., 51: 1-39. DOI: $10.1111 / 1467-8586.00069$

Yang, X.S., 2008. Mathematical Modelling for Earth Sciences. 1st Edn., Dunedin Academic Press, UK. 